\title{
Data Storage
}

National Cancer Institute

\section{Source}

National Cancer Institute. Data Storage. NCI Thesaurus. Code C142494.

To maintain data by placing the data, or a copy of the data, onto an electronically accessible device for preservation (either in plain-text or encrypted format). (HL7) 